\title{
Transport in Anisotropic Superfluids: A Holographic Description
}

\author{
Johanna Erdmenger \\ Max-Planck-Institut für Physik, Munich \\ E-mail: jke@mpp.mpg.de \\ Daniel Fernández * \\ Departament de Física Fonamental \& Institut de Ciències del Cosmos (ICC), Universitat de \\ Barcelona $(U B)$ \\ E-mail: danieleffn.ub.edu
}

Patrick Kerner

Max-Planck-Institut für Physik, Munich

E-mail: pkerner@mpp.mpg.de

\section{Hansjörg Zeller}

Max-Planck-Institut für Physik, Munich

E-mail: zeller@mpp.mpg.de

\begin{abstract}
We study transport phenomena in p-wave superfluids in the context of gauge/gravity duality. Due to the spacetime anisotropy of this system, the tensorial structure of the transport coefficients is non-trivial in contrast to the isotropic case. In particular, there is an additional shear mode which leads to a non-universal value of the shear viscosity even in an Einstein gravity setup. In this proceeding, we present a study of the helicity two, helicity one and parts of the helicity zero fluctuation modes. In addition to the non-universal shear viscosity, we also investigate the thermoelectric effect, i.e. the mixing of electric and heat current. Moreover, we also find an additional effect due to the anisotropy, the so-called flexoelectric effect.
\end{abstract}

Xth Quark Confinement and the Hadron Spectrum

8 - 12 October 2012

TUM Campus Garching, Munich, Germany

*DF acknowledges financial support from 2009-SGR-168, MEC FPA2010-20807-C02-01, MEC FPA2010-20807C02-02, CPAN CSD2007-00042 Consolider-Ingenio 2010, and ERC StG 306605 HoloLHC.

$\dagger$ Speaker. 


\section{Introduction}

Hydrodynamics is a very powerful description of systems close to equilibrium. Its focus is on slowly varying fluctuations with frequency $\omega$ and momentum $k$ smaller than the typical length scale, the mean free path. Hydrodynamics may be seen as the low-energy effective description of interacting systems. Gauge/gravity duality is a very useful tool to further develop the hydrodynamic description for various systems.

Here we concentrate on an example of an anisotropic system, the so called backreacted p-wave superfluid. We start with an Einstein-Yang-Mills action and look at black hole solutions at finite isospin density. It turns out that depending on the choice of parameters, more precisely on the ratio of temperature to chemical potential $T / \mu$, we find the well known AdS Reissner-Nordström black hole solution or a black hole solution with vector hair. The latter spontaneously breaks the $U(1)$ symmetry due to the chemical potential and the $S O(3)$ rotational symmetry. This can be related to an anisotropic fluid on the field theory side in which the transport coefficients depend on the direction, which means they are tensors. In the case we study here the fluid is transversely symmetric, i.e. the system has an $S O(2)$ symmetry and we can use this symmetry to reduce the tensors to the minimal amount of independent quantities. For instance, the viscosity which relates the stress $T^{\mu v}$ in a fluid with the strain $\nabla_{\lambda} u_{\rho}+\nabla_{\rho} u_{\lambda}$ given in terms of the four velocity of the fluid $u_{\mu}$ is parametrized by a rank four tensor $\eta^{\mu \nu \lambda \rho}$ (see [1]). Using the symmetry we find two independent components of the shear viscosity tensor, in contrast to only one in the isotropic case ( $S O$ (3) symmetry). Due to the symmetries of our system it turns out that one of the strains related to one of the shear viscosity components transforms as a tensor under the remaining $S O(2)$ symmetry while the other one transforms as a vector. This will turn out to be very important for the results we show in this proceeding.

A very famous result in the context of gauge/gravity duality is that the ratio between shear viscosity and the entropy density is universal [2-4]. The ratio is the same for all field theories which have an Einstein gravity dual, which means that the field theory is a large $N$ gauge theory at infinite 't Hooft coupling $\lambda$. In [5] and [1] we have shown that universality is absent even at leading order in $N$ and $\lambda$ if the fluid is anisotropic. In this case, the universality is lost since one of the different shear modes transforms as a helicity one mode under the rotational symmetry and can therefore couple to other helicity one modes present in the system. The coupling generates nontrivial dynamics which lead to a non-universal behavior of the shear viscosity. This result is valid for a field theory dual to Einstein gravity without additional contributions to the gravity action. The detailed calculations can be found in [1].

Among the remaining helicity one and helicity zero modes we find some additional transport phenomena: the thermoelectric effect in the transverse direction (helicity one) as well as in the direction parallel to the condensate (helicity zero) and the flexoelectric effect (helicity one). For a detailed study of the helicity one and zero modes see $[1,6]$. The thermoelectric effect is the phenomenon that the electric and heat current mix since charged objects transport charge as well as energy. The flexoelectric effect is known from nematic liquids which consist of molecules with non-zero dipole moment (see e.g. [7]). A direction can be preferred by the dipoles. In this anisotropic phase, a strain can lead to effective polarization of the liquid and an electric field applied to the liquid can lead to a stress. 


\section{Background}

For a detailed study of the setup, we use, see [1,8]. Here we give a brief review of its most important properties. We consider $S U(2)$ Einstein-Yang-Mills theory in $(4+1)$-dimensional asymptotically AdS space. The action is

$$
S=\int \mathrm{d}^{5} x \sqrt{-g}\left[\frac{1}{2 \kappa_{5}^{2}}(R-\Lambda)-\frac{1}{4 \hat{g}^{2}} F_{M N}^{a} F^{a M N}\right]+S_{\mathrm{bdy}},
$$

where $\kappa_{5}$ is the five-dimensional gravitational constant, $\Lambda=-\frac{12}{L^{2}}$ is the cosmological constant (with $L$ being the $A d S$ radius), and $\hat{g}$ is the Yang-Mills coupling constant. It is convenient to define $\alpha \equiv \frac{\kappa_{5}}{\hat{g}}$, which measures the strength of the backreaction.

To solve the Einstein and Yang-Mills equations, we use the following ansätze for the gauge field and the metric, which can be motivated from symmetry considerations $[8,9]$

$$
\begin{gathered}
A=\phi(r) \tau^{3} \mathrm{~d} t+w(r) \tau^{1} \mathrm{~d} x, \\
\mathrm{~d} s^{2}=-N(r) \sigma(r)^{2} \mathrm{~d} t^{2}+\frac{1}{N(r)} \mathrm{d} r^{2}+r^{2} f(r)^{-4} \mathrm{~d} x^{2}+r^{2} f(r)^{2}\left(\mathrm{~d} y^{2}+\mathrm{d} z^{2}\right),
\end{gathered}
$$

where $N(r) \equiv-\frac{2 m(r)}{r^{2}}+\frac{r^{2}}{L^{2}}$. The $A d S$ boundary is at $r \rightarrow \infty$ and for our black hole solutions we denote the position of the horizon as $r_{h}$.

This ansatz is compatible with the well-known AdS Reissner-Nordström solution with $w(r)=$ 0 for all values of $r$. It preserves the $S O(3)$ symmetry and corresponds to the normal phase of the system. There is a second solution with non-vanishing $w(r)$, which can only be computed numerically. The second solution breaks the rotational $S O(3)$ symmetry and describes the condensed superfluid phase. Due to our choice of boundary conditions, this breaking occurs spontaneously.

We express field theory quantities in terms of the boundary values of the fields introduced above. The temperature $T$ and the entropy density $s$ are given by the values of the fields at the horizon. From the values at the AdS boundary we identify, using the AdS/CFT dictionary, the expectation values of the dual operators,

$$
\left\langle\mathscr{J}_{3}^{t}\right\rangle,\left\langle\mathscr{J}_{1}^{x}\right\rangle,\left\langle\mathscr{T}_{t t}\right\rangle,\left\langle\mathscr{T}_{x x}\right\rangle \text { and }\left\langle\mathscr{T}_{y y}\right\rangle=\left\langle\mathscr{T}_{z z}\right\rangle
$$

In [8] it was found that the value of the Yang-Mills coupling constant $\alpha$ determines whether the phase transition between the two solutions described above is second order ( $\alpha \leq \alpha_{c}=0.365$ ) or first order $\left(\alpha>\alpha_{c}=0.365\right)$. For values above a certain $T_{c} / \mu$ the thermodynamically preferred solution is the Reissner-Nordström black hole, below this value the solution with a condensate corresponds to the ground state. For a detailed discussion see [8].

\section{Fluctuations and Transport Properties}

In this section we present the transport found in the holographic p-wave superfluid under small perturbations. We split our analysis into distinct transport phenomena. On the gravity side, the perturbations are given by fluctuations of the metric $h_{M N}\left(x^{\mu}, r\right)$ and the gauge field $a_{M}^{a}\left(x^{\mu}, r\right)$. Thus we are studying a total of 14 physical modes: 5 coming from the massless graviton in 5 dimensions and 


\begin{tabular}{c|ccc} 
& dynamical fields & constraints & \# physical modes \\
\hline helicity 2 & $h_{y z}, h_{y y}-h_{z z}$ & none & 2 \\
helicity 1 & $h_{t y}, h_{x y} ; a_{y}^{a}$ & $h_{y r}$ & 4 \\
& $h_{t z}, h_{x z} ; a_{z}^{a}$ & $h_{z r}$ & 4 \\
helicity 0 & $h_{t t}, h_{x x}, h_{y y}+h_{z z}, h_{x t} ; a_{t}^{a}, a_{x}^{a}$ & $h_{t r}, h_{x r}, h_{r r} ; a_{r}^{a}$ & 4
\end{tabular}

Table 1: Classifications of the fluctuations according to their transformation under the little group $S O(2)$. The constraints are given by the equations of motion for the fields which are set to zero due the fixing of the gauge freedom: $a_{r}^{a} \equiv 0$ and $h_{r M} \equiv 0$. The number of physical modes is obtained by the number of dynamical fields minus the number of constraints. Due to $S O(2)$ invariance the fields in the first and second line of the helicity one fields can be identified.

$3 \times 3$ from the massless vectors in five dimensions. Due to time and spatial translation invariance in the Minkowski directions, the fluctuations can be decomposed in a Fourier decomposition as

$$
h_{M N}\left(x^{\mu}, r\right)=\int \frac{\mathrm{d}^{4} k}{(2 \pi)^{4}} \mathrm{e}^{\mathrm{i} k_{\mu} x^{\mu}} \hat{h}_{M N}\left(k^{\mu}, r\right) \text { and } a_{M}^{a}\left(x^{\mu}, r\right)=\int \frac{\mathrm{d}^{4} k}{(2 \pi)^{4}} \mathrm{e}^{\mathrm{i} k_{\mu} x^{\mu}} \hat{a}_{M}^{a}\left(k^{\mu}, r\right) \text {. }
$$

In the following we also set the spatial momentum $\vec{k}=0$. Thus we can classify the fluctuations under the remaining $S O(2)$ rotational symmetry still present in our system (see table 1).

\subsection{Thermoelectric Effect}

In the system at hand we find 2 thermoelectric effects, one perpendicular to the condensate (helicity one) and one parallel to the condensate (helicity zero). The coupling between thermal and electrical transport is well known in condensed matter physics, since the charge carriers (electron or holes) transport charge as well as heat. The fields on the gravity side related to this effect are $a_{\perp}^{3}$ and $h_{t \perp}$ in the transverse direction and $a_{x}^{3}$ and $h_{t x}$ in the parallel direction. Note that two more fields, $a_{t}^{1}$ and $a_{t}^{2}$ couple to $a_{x}^{3}$ in the latter case. However, in this proceeding we are not interested in these couplings, for a discussion on the matter see [6].

We begin with the well known connection between electrical $\left\langle J_{3}^{\perp / x}\right\rangle=\left\langle J^{\perp / x}\right\rangle$ and thermal $\left\langle Q^{\perp / x}\right\rangle=\left\langle T^{t \perp / t x}\right\rangle-\mu\left\langle J^{\perp / x}\right\rangle$ transport $^{1}$, i.e.

$$
\left(\begin{array}{c}
\left\langle J^{\perp / x}\right\rangle \\
\left\langle Q^{\perp / x}\right\rangle
\end{array}\right)=\left(\begin{array}{cc}
\sigma^{\perp \perp / x x} & T \alpha^{\perp \perp / x x} \\
T \alpha^{\perp \perp / x x} & T \bar{\kappa}^{\perp \perp / x x}
\end{array}\right)\left(\begin{array}{c}
E_{\perp / x} \\
-\left(\nabla_{\perp / x} T\right) / T
\end{array}\right),
$$

where the electric field $E_{\perp / x}$ and the thermal gradient $-\nabla_{\perp / x} T / T$ are related to the boundary values of the gauge field $a_{\perp / x}^{3}$ and the metric $h_{t \perp / t x}$ (For the precise relation see [1,6]). Putting all together and comparing the relation of the electric and thermal transport to the corresponding quantities on the gravity side, we can identify the transport matrix of (3.2),

$$
\sigma=-\frac{\mathrm{i} G_{3,3}}{\omega}, T \alpha=\frac{\mathrm{i}}{\omega}\left\langle\mathscr{J}_{3}^{t}\right\rangle-\mu \sigma, T \bar{\kappa}=\frac{\mathrm{i}}{\omega}\left(\left\langle\mathscr{T}_{t t}\right\rangle-2 \mu\left\langle\mathscr{J}_{3}^{t}\right\rangle\right)+\mu^{2} \sigma .
$$

Note that these equations are valid for both directions, parallel and transverse to the condensate.

\footnotetext{
${ }^{1}$ We use curly notation $(\mathscr{J}, \mathscr{T})$ for the currents in thermal equilibrium and normal letters $(J, T)$ for currents including the dissipative terms.
} 


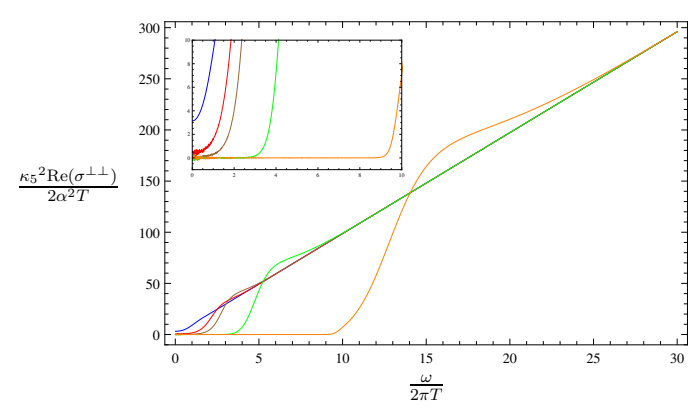

(a) color coding: blue $T=\infty$, red $T=1.00 T_{c}$, brown $T=0.88 T_{c}$, green $T=0.50 T_{c}$, orange $T=0.19 T_{c}$

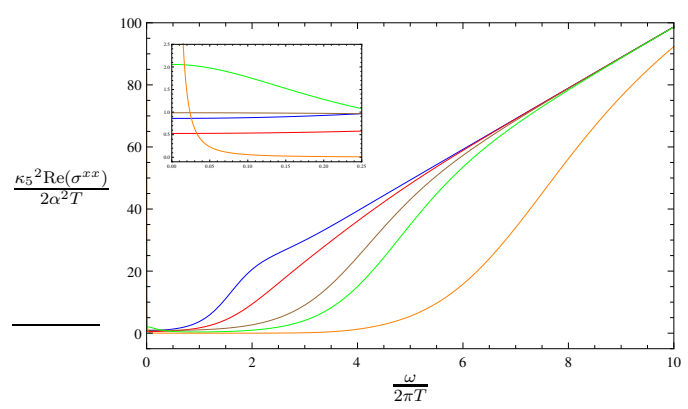

(b) color coding: blue $T=1.63 T_{c}$, red $T=0.98 T_{c}$, brown $T=0.88 T_{c}$, green $T=0.78 T_{c}$, orange $T=$ $0.50 T_{c}$

Figure 1: Real part of the conductivity $\operatorname{Re}\left(\sigma^{\perp \perp / x x}\right)$ over the frequency $\omega /(2 \pi T)$ for $\alpha=0.316$. For a description and comparison of the plots see text below.

In figure 1 we plot our numerical results for $\operatorname{Re}\left(\sigma^{\perp \perp / x x}\right)$ versus the frequency $\omega /(2 \pi T)$ for $\alpha=0.316 \lesssim \alpha_{c}$. For large frequencies, i. e. $\omega \gg 2 \pi T$, in both cases the conductivity asymptotically has a linear dependence on the frequency, i.e. $\operatorname{Re}\left(\sigma^{\perp \perp / x x}\right) \sim \omega$ for $\omega \gg 2 \pi T$.

For small temperatures $\left(T<T_{c}\right)$ we see a gap opening up at small frequencies. The size of the gap increases as the temperature is decreased. This is the expected energy gap we know from superconductors. The gap ends at a frequency $\omega_{g}$ with a sharp increase of the conductivity transverse to the condensate. In comparison, the increase with the frequency in the case parallel to the condensate is more shallow. Beyond the gap the conductivity transverse to the condensate at small temperature $\left(T<T_{c}\right)$ is larger than the corresponding value at large temperature $(T>$ $T_{c}$ ) such that the small temperature conductivities approach the asymptotic behavior from above. This is opposed to the parallel case where the asymptotic value is approached from below for all temperatures.

The value of $\operatorname{Re}\left(\sigma^{\perp \perp}\right)$ at $\omega=0$ approaches zero with decreasing temperature. Below $T_{c}$ the tendency for this part of the conductivity to vanish increases. Nevertheless, we still find finite values even below $T_{c}$, i.e. these values seem to be suppressed but not identically vanishing (c.f. [10]). Finally, we observe that an increase in $\alpha$ leads to a stronger suppression of the real part of the conductivity in the gap region.

The real part of $\sigma^{x x}$, as opposed to the perpendicular case, increases again for small but finite frequencies and reaches a finite value in the $\omega \rightarrow 0$ limit, as seen in the zoomed region of figure 1(b). This increase in the real part in the zero frequency limit is due to a quasinormal mode which moves up the imaginary axis in the complex frequency plane and seems to reach the origin $\omega=0$ at temperatures slightly above $0.5 T_{c}$. The increase we see towards the $\omega \rightarrow 0$ limit comes from the projection of the quasinormal mode onto the real frequency axis. Note that this bump increases with decreasing temperature. Unfortunately it is challenging to compute the exact temperature at which the mode reaches the origin, since we have to rely on numerical calculations. Nevertheless, for temperatures below $0.5 T_{c}$ it appears that a pole in the real part of the conductivity is formed and at the same time the real part of the conductivity gets more strongly suppressed at finite small frequencies in comparison to cases of temperatures above $0.5 T_{c}$ (see the green and orange curve in 
the zoomed region of figure $1(\mathrm{~b})$ ). It seems that somewhere around $0.5 T_{c}$, due to the quasinormal mode at the origin, the conductivity behavior in the direction of the condensate changes.

Due to the sum rule for the conductivity, i.e. the frequency integral over the real part of the conductivity is constant for all temperatures, a delta peak has to form at zero frequency which contains the "missing area" of the gap region. The strength of the delta peak has two contributions: the first is proportional to the superfluid density $n_{s}^{\perp / x}, \operatorname{Re}\left(\sigma^{\perp \perp / x x}\right) \sim \alpha^{2} / \kappa_{5}^{2} \pi n_{s}^{\perp / x} \delta(\omega)$ and appears only for temperatures below $T_{c}$. The second contribution, the Drude peak, is a consequence of translation invariance of our system and appears for all temperatures.

The delta peak is observed in the imaginary part of the conductivity by using the KramersKronig relation (see [10]),

$$
\operatorname{Im}\left(\sigma^{\perp \perp / x x}\right) \simeq \frac{A_{D}^{\perp / x}(\alpha, T)}{\omega}+\frac{A_{s}^{\perp / x}(\alpha)}{\omega}\left(1-\frac{T}{T_{c}}\right)
$$

for $T \lesssim T_{c}$, with $A_{s}(\alpha)\left(1-\frac{T}{T_{c}}\right) \propto n_{s}$ and $A_{D}$ parametrizing the contribution from the Drude peak. We see both contributions in both cases, however, with different magnitudes, e.g. by plotting $\omega \operatorname{Im}\left(\sigma^{\perp \perp / x x}\right)($ see $[1,6])$.

\subsection{Non-Universal Shear Viscosity and Flexoelectric Effect}

In this section we present the transport generated by the remaining three components of the helicity one modes, $a_{\perp}^{ \pm}=a_{\perp}^{1} \pm i a_{\perp}^{2}$ and $h_{x \perp}$ which are related to the field theory expectation values $\left\langle J_{ \pm}^{\perp}\right\rangle$ and $\left\langle T^{x \perp}\right\rangle$. We first focus on $\left\langle T^{x \perp}\right\rangle$, which for $\left(a_{\perp}^{ \pm}\right)_{0}^{b}=0$ can be translated into the following dual field theory behavior

$$
\left\langle T^{x \perp}\right\rangle=-\left\langle\mathscr{T}_{x x}\right\rangle h_{x \perp}-i \omega \eta_{x \perp} h_{x \perp},
$$

where $\eta_{x \perp}$ is the second shear viscosity which is present in a transverse isotropic fluid and with $\left\langle\mathscr{T}_{x x}\right\rangle$ as defined in (2.4). Here we see that we can apply the Kubo formula to determine the shear viscosity $\eta_{x \perp}$,

$$
\eta_{x \perp}=-\lim _{\omega \rightarrow 0} \frac{1}{\omega} \operatorname{Im}\left(G^{x \perp, x \perp}\right) .
$$

As described in $[1,5]$, this shear viscosity has a non-trivial temperature dependence even in the large $N$ and large $\lambda$ limit and is therefore not universal. In fig. 2 we compare our numerical results for the ratio of the shear viscosity $\eta_{x \perp}$ to the entropy density $s$ with the universal behavior ${ }^{2}$ of the shear viscosity $\eta_{y z}$ for different values of $\alpha$. We see that in the normal phase $T \geq T_{c}$, the two shear viscosity components coincide as required in an isotropic fluid. In addition, the ratio of shear viscosity to entropy density is universal. In the superfluid phase $T<T_{c}$, the two components of the shear viscosity tensor deviate from each other and $\eta_{x \perp}$ is non-universal. However it is exciting that $\eta_{x \perp} / s \geq 1 / 4 \pi$, such that the KSS bound on the ratio of shear viscosity to entropy density [2] is still valid.

The difference between the two viscosity components in the superfluid phase is controlled by $\alpha$ as defined in section 2. In the probe limit where $\alpha=0$, the shear viscosities also coincide in the

\footnotetext{
${ }^{2} \eta_{y z}$ is generated by the minimally coupled scalar field $h_{z}^{y}$. Therefore $\eta_{y z} / s=1 / 4 \pi$ for all temperatures and values of $\alpha$ (see [4]).
} 


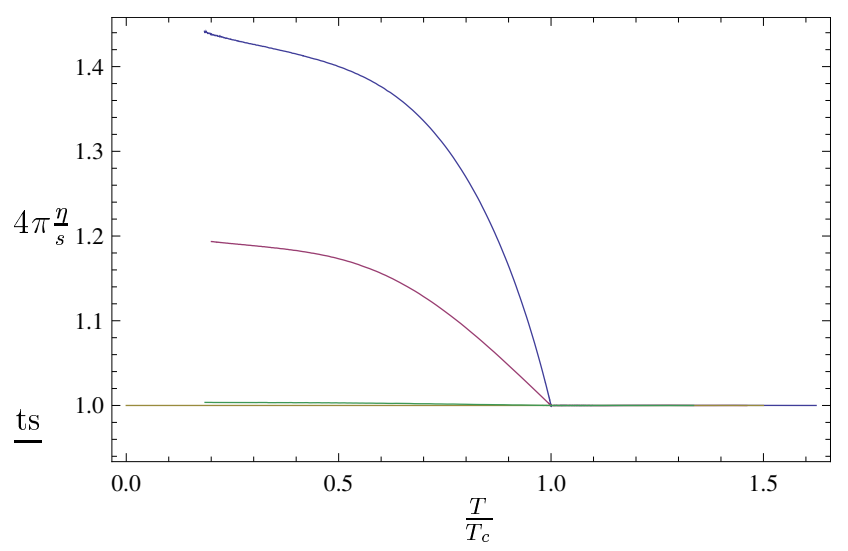

Figure 2: Ratio of shear viscosities $\eta_{y z}$ and $\eta_{x \perp}$ to entropy density $s$ over the reduced temperature $T / T_{c}$ for different values of the ratio of the gravitational coupling constant to the Yang-Mills coupling constant $\alpha$. The color coding is as follows: In yellow, $\eta_{y z} / s$ for all values of $\alpha$; while the curve for $\eta_{x \perp} / s$ is plotted in green for $\alpha=0.032$, red for $\alpha=0.224$ and blue for $\alpha=0.316$.

superfluid phase. By increasing the backreaction of the gauge fields, i. e. rising $\alpha$, the deviation between the shear viscosities becomes larger in the superfluid phase as shown in fig. 2. If $\alpha$ is larger than the critical value $\alpha_{c}=0.365$ found in [8] where the phase transition to the superfluid phase becomes first order, the shear viscosity components are also multivalued close to the phase transition (see [1]). There is a maximal $\alpha$ denoted by $\alpha_{\max }=0.395$ for which the superfluid phase exists, thus we expect that the deviation of the shear viscosity $\eta_{x \perp}$ from its universal value is maximal for this $\alpha_{\max }$. Unfortunately numerical calculations for large values of $\alpha$ are very challenging such that we cannot present satisfying numerical data for this region.

As described in our letter [5], we also have found numerically that for $\alpha<\alpha_{c}$

$$
1-4 \pi \frac{\eta_{x \perp}}{s} \propto\left(1-\frac{T}{T_{c}}\right)^{\beta} \quad \text { with } \quad \beta=1.00 \pm 3 \% .
$$

Interestingly, the value of $\beta$ appears to be independent of $\alpha$. This result has been confirmed by an analytic calculation in [11].

The non-universality of this component of the shear viscosity is due to the coupling between $h_{x \perp}$ and $a_{\perp}^{1}$, which does not vanish in the $\omega \rightarrow 0$ limit. This is in contrast to the universal case generated by the fluctuation $h_{y z}$. Note that the coupling depends on the condensate $w$ and therefore vanishes in the unbroken case. Hence, as we confirm numerically in fig. 2, in the unbroken case $\left(T>T_{c}\right)$ we obtain for both components the universal result.

In addition to the flavor conductivity and the shear viscosity we obtain a coupling between the stress $\left\langle T^{x \perp}\right\rangle$ and the flavor fields $a_{\perp}^{ \pm}$as well as the currents $\left\langle J_{ \pm}^{\perp}\right\rangle$ and the strain $h_{x \perp}$. This coupling introduces an effect which is called flexoelectric effect in nematic crystals [7] and only appears in fluids with broken rotational symmetry. We have a current $\left\langle\mathscr{J}_{1}^{x}\right\rangle$ in a favored direction in the background which interacts with the flavor fields $a_{\perp}^{ \pm}$. This interaction induces a force on the current which pushes the current in its perpendicular direction generating the stress $\left\langle T^{x \perp}\right\rangle$. In the similar way, a strain $h_{x \perp}$ introduces an inhomogeneity in the current $\left\langle\mathscr{J}_{1}^{x}\right\rangle$ resulting in a flavor field $a_{\perp}^{ \pm}$ which generates the currents $\left\langle J_{ \pm}^{\perp}\right\rangle$. For a detailed discussion see [1]. 


\section{Conclusion}

We have presented some of the transport properties found in a holographic p-wave superfluid with backreaction. It turns out that due to the backreaction and the breaking of the rotational symmetry many different transport phenomena can be studied. For instance, we have shown that one of the two shear viscosity components when divided by the entropy density displays a temperature dependence, whereas the other component is constant and takes the well known universal value of $1 / 4 \pi$. Note that the departure from the universal value is found at leading order in large $N$ and large 't Hooft coupling $\lambda$. Furthermore the differences between the thermoelectric effect in direction transverse and perpendicular to the condensate were presented. Finally, we have seen an effect similar to the flexoelectric effect, i.e. coupling between a strain and an electric field. However, in our case we have a coupling between a strain and flavor fields. For a detailed analysis of the effects presented here see $[1,5,6]$.

\section{Acknowledgements}

This work was supported in part by The Cluster of Excellence for Fundamental Physics Origin and Structure of the Universe.

\section{References}

[1] J. Erdmenger, P. Kerner, and H. Zeller, Transport in Anisotropic Superfluids: A Holographic Description, JHEP 1201 (2012) 059, [arXiv: 1110 .0007].

[2] P. Kovtun, D. T. Son, and A. O. Starinets, Viscosity in strongly interacting quantum field theories from black hole physics, Phys. Rev. Lett. 94 (2005) 111601, [hep-th/ 0405231 ].

[3] A. Buchel and J. T. Liu, Universality of the shear viscosity in supergravity, Phys. Rev. Lett. 93 (2004) 090602, [hep-th/0311175].

[4] N. Iqbal and H. Liu, Universality of the hydrodynamic limit in AdS/CFT and the membrane paradigm, Phys. Rev. D79 (2009) 025023, [arXiv:0809.3808].

[5] J. Erdmenger, P. Kerner, and H. Zeller, Non-universal shear viscosity from Einstein gravity, Phys.Lett. B699 (2011) 301-304, [arXiv: 1011 . 5912].

[6] J. Erdmenger, D. Fernandez, and H. Zeller, New Transport Properties of Anisotropic Holographic Superfluids, arXiv:1212.4838.

[7] P. de Gennes, The Physics of Liquid Crystals. Oxford University Press, 1974.

[8] M. Ammon, J. Erdmenger, V. Grass, P. Kerner, and A. O'Bannon, On Holographic p-wave Superfluids with Back-reaction, Phys. Lett. B686 (2010) 192-198, [arXiv: 0912 . 3515].

[9] S. S. Gubser and S. S. Pufu, The gravity dual of a p-wave superconductor, JHEP 11 (2008) 033, [arXiv:0805.2960].

[10] S. A. Hartnoll, C. P. Herzog, and G. T. Horowitz, Holographic Superconductors, JHEP 12 (2008) 015, [arXiv:0810.1563].

[11] P. Basu and J.-H. Oh, Analytic Approaches to An-Isotropic Holographic Superfluids, arXiv:1109.4592. 